\title{
Upper bounds on the compactness at the innermost light ring of anisotropic horizonless spheres
}

\author{
Yan Peng ${ }^{\mathrm{a}}$ \\ School of Mathematical Sciences, Qufu Normal University, Qufu 273165, Shandong, China
}

Received: 14 July 2020 / Accepted: 14 August 2020 / Published online: 19 August 2020

(C) The Author(s) 2020

\begin{abstract}
In the background of isotropic horizonless spheres, Hod recently provided an analytical proof of a bound on the compactness at the innermost light ring with the dominant energy condition. In this work, we extend the discussion of isotropic spheres to anisotropic spheres. With the dominant energy and non-negative trace conditions, we prove that Hod's bound also holds in the case of anisotropic horizonless spheres.
\end{abstract}

\section{Introduction}

According to general relativity, highly curved spacetimes may possess closed light rings (null circular geodesics), on which massless particles can orbit in a circle [1,2]. It is well known that closed light rings are usually related to black hole spacetimes. In fact, closed light rings may also exist in the horizonless ultra-compact spacetime. From theoretical and astrophysical aspects, the light rings have been extensively studied in various gravity backgrounds [3-13].

The closed light ring plays an important role in understanding properties of curved spacetimes. For example, the interesting phenomenon of strong gravitational lensing in highly curved spacetimes is closely related to the existence of light rings [14]. In addition, the light ring can be used to describe the distribution of exterior matter fields outside black holes [15-20]. And it was also proved that the innermost light ring provides the fastest way to circle a central black hole as measured by observers at the infinity [21-23]. Moreover, the existence of stable light rings suggests that the central compact stars may suffer from nonlinear instabilities [24-30]. And unstable light rings can be used to determine the characteristic resonances of black holes [31-38].

Recently, the compactness at the innermost closed light ring was investigated. The compactness can be described by the parameter $\frac{m(r)}{r}$, where $m(r)$ is the gravitational mass

a e-mail: yanpengphy@ 163.com (corresponding author) within the radius $r$. In the case of black holes, the compactness parameter at the innermost light ring is characterized by the lower bound $\frac{m\left(r_{\gamma}^{i n}\right)}{\left.r_{\gamma}^{i n}\right)} \geqslant \frac{1}{3}$ with $r_{\gamma}^{i n}$ as the innermost light ring radius. However, very differently in the horizonless case, numerical data in [39] suggests that the compactness parameter may satisfy an upper bound $\frac{m\left(r_{\gamma}^{i n}\right)}{\left.r_{\gamma}^{i n}\right)} \leqslant \frac{1}{3}$ for spherically symmetric ultra-compact isotropic spheres. Hod has provided compact analytical proofs of the characteristic intriguing bound $\frac{m\left(r_{\gamma}^{i n}\right)}{\left.r_{\gamma}^{i n}\right)} \leqslant \frac{1}{3}$ for the spherically symmetric spatially regular spheres with isotropic tensor $\left(p=p_{\tau}\right)$, where $p$ and $p_{\tau}$ are interpreted as the radial pressure and the tangential pressure respectively [40].

In the present paper, we study the compactness at the innermost light ring of horizonless spheres. We shall prove the bound $\frac{m\left(r_{\gamma}^{i n}\right)}{\left.r_{\gamma}^{i n}\right)} \leqslant \frac{1}{3}$ in the case of anisotropic sphere with $p \neq p_{\tau}$. We point out that this bound in the isotropic case of $p=p_{\tau}$ has been proved in [40]. Our main results are included in the last section.

\section{Investigations on the compactness at the innermost light ring}

We study the closed light ring of spherically symmetric configurations. In the standard Schwarzschild coordinate, these spacetimes are expressed by line element [40]

$d s^{2}=-e^{-2 \delta} \mu d t^{2}+\mu^{-1} d r^{2}+r^{2}\left(d \theta^{2}+\sin ^{2} \theta d \phi^{2}\right)$,

where the metric has two functions $\delta(r)$ and $\mu(r)=1-$ $\frac{2 m(r)}{r}$. For horizonless asymptotically flat spacetimes, the metric functions are characterized by the near origin behavior [40-42]

$\mu(r \rightarrow 0)=1+O\left(r^{2}\right) \quad$ and $\quad \delta(0)<\infty$ 
and the far region behavior [40-42]

$\mu(r \rightarrow \infty)=1 \quad$ and $\quad \delta(r \rightarrow \infty)=0$.

We denote the components of the energy-momentum tensor as

$\rho=-T_{t}^{t}, \quad p=T_{r}^{r} \quad$ and $\quad p_{\tau}=T_{\theta}^{\theta}=T_{\phi}^{\phi}$,

where $\rho, p$ and $p_{\tau}$ are respectively the energy density, the radial pressure and the tangential pressure of the horizonless configurations $[17,43]$. For the case of isotropic energymomentum tensor, there is the relation $p=p_{\tau}$ [40]. In this work, our discussion also covers the case of $p \neq p_{\tau}$. The unknown metric functions are determined by the Einstein equations $G_{v}^{\mu}=8 \pi T_{v}^{\mu}$. With the energy density and pressures (4), one can express the Einstein field equations in the form

$\mu^{\prime}=-8 \pi r \rho+\frac{1-\mu}{r}$,

$\delta^{\prime}=\frac{-4 \pi r(\rho+p)}{\mu}$.

Using the Einstein field Eqs. (5) and (6), it has been explicitly proved that the closed light rings are characterized by the relation [41]

$\mathcal{R}(r)=3 \mu(r)-1-8 \pi r^{2} p(r)=0 \quad$ for $\quad r=r_{\gamma}$,

where $r_{\gamma}$ is the radius of the closed light ring.

From Eq. (2) and the regular condition $p(0)<\infty$, the function $\mathcal{R}(r)$ is characterized by asymptotical behaviors

$\mathcal{R}(r)=3 \mu(r)-1-8 \pi r^{2} p(r) \rightarrow 2$ for $r \rightarrow 0$.

We label $r_{\gamma}^{i n}$ as the radius of the innermost closed light ring, which corresponds to the smallest positive root of $\mathcal{R}(r)=0$. In the range $\left[0, r_{\gamma}^{i n}\right]$, the function $\mathcal{R}(r)$ satisfies the relation

$\mathcal{R}(r) \geqslant 0 \quad$ for $\quad r \in\left[0, r_{\gamma}^{i n}\right]$

In particular, at the innermost closed light ring, there is the relation

$\mathcal{R}(r)=3 \mu(r)-1-8 \pi r^{2} p(r)=0 \quad$ for $\quad r=r_{\gamma}^{i n}$.

Substituting Eqs. (5) and (6) into the conservation equation $T_{r ; \mu}^{\mu}=0$, one obtains a relation

$P^{\prime}(r)=\frac{r}{2 g}[\mathcal{R}(\rho+p)+2 \mu T]$,

where $P(r)=r^{4} p(r), \mathcal{R}=3 \mu-1-8 \pi r^{2} p$ and $T=$ $-\rho+p+2 p_{\tau}$.

In proving the following bound (22), Hod has imposed the dominant energy condition [40]. In this work, we impose the same dominant energy condition

$\rho \geqslant|p|,\left|p_{\tau}\right| \geqslant 0$.
And we also take the non-negative trace condition, which is [39-42]

$T=-\rho+p+2 p_{\tau} \geqslant 0$.

In fact, for a polytropic pressure density equation of the form $p=p_{\tau}=k_{p} \rho$, Hod has obtained a bound $\frac{m\left(r_{\gamma}^{i n}\right)}{\left.r_{\gamma}^{i n}\right)} \leqslant$ $\frac{k_{p}+2}{6\left(k_{p}+1\right)}$, where $\rho, p$ and $p_{\tau}$ are interpreted as the energy density, the radial pressure and the tangential pressure respectively [40]. In the case of $k_{p} \geqslant \frac{1}{3}$, Hod's bound is $\frac{m\left(r_{\gamma}^{i n}\right)}{\left.r_{\gamma}^{i n}\right)} \leqslant \frac{1}{3}$, which is the same as (22). So Hod proved the bound (22) in the case of $p=p_{\tau}$. In the present work, we generalize the discussion to cover the case of $p \neq p_{\tau}$.

Relations (9-13) yield that the function $P(r)$ satisfies the inequality

$P^{\prime}(r) \geqslant 0 \quad$ for $\quad r \in\left[0, r_{\gamma}^{i n}\right]$.

Near the origin, the pressure function $P(r)$ has the asymptotical behavior

$P(r \rightarrow 0)=0$

With relations (14) and (15), one obtains

$P(r) \geqslant 0 \quad$ for $\quad r \in\left[0, r_{\gamma}^{i n}\right]$.

The relation (16) and $P(r)=r^{4} p(r)$ yield that

$p(r) \geqslant 0 \quad$ for $\quad r \in\left[0, r_{\gamma}^{i n}\right]$.

In particular, at the innermost light ring, there is the relation

$p\left(r_{\gamma}^{i n}\right) \geqslant 0$

According to (10) and (18), one finds that

$3 \mu(r)-1=8 \pi r^{2} p(r) \geqslant 0 \quad$ for $\quad r={ }_{\gamma}^{i n}$.

The relation (19) yields that

$\mu\left(r_{\gamma}^{i n}\right) \geqslant \frac{1}{3}$

The inequality (20) can be expressed as

$1-\frac{2 m\left(r_{\gamma}^{i n}\right)}{r_{\gamma}^{i n}} \geqslant \frac{1}{3}$.

Then we obtain an upper bound on the compactness at the innermost light ring

$\frac{m\left(r_{\gamma}^{i n}\right)}{r_{\gamma}^{i n}} \leqslant \frac{1}{3}$. 


\section{Conclusions}

We studied the compactness at the innermost light ring of anisotropic horizonless spheres. We assumed the dominant energy and non-negative trace conditions. At the innermost light ring, we obtained an upper bound on the compactness expressed as $\frac{m\left(r_{\gamma}^{i n}\right)}{r_{\gamma}^{i n}} \leqslant \frac{1}{3}$, where $r_{\gamma}^{i n}$ is the innermost light ring and $m\left(r_{\gamma}^{i n}\right)$ is the gravitational mass within the sphere of radius $r_{\gamma}^{i n}$. In fact, Hod firstly proved this bound in the spacetime of horizonless spheres with isotropic tensor $p=$ $p_{\tau}$ [40]. In the present work, we proved the same bound in the background of horizonless spheres with generalized anisotropic tensor covering the case of $p \neq p_{\tau}$.

Acknowledgements We would like to thank the anonymous referee for the constructive suggestions to improve the manuscript. This work was supported by the Shandong Provincial Natural Science Foundation of China under Grant No. ZR2018QA008. This work was also supported by a grant from Qufu Normal University of China under Grant No. xkjjc201906.

Data Availability Statement This manuscript has no associated data or the data will not be deposited. [Authors' comment: I would like to emphasize that all relevant physical and mathematical calculations are explicitly presented in this paper.]

Open Access This article is licensed under a Creative Commons Attribution 4.0 International License, which permits use, sharing, adaptation, distribution and reproduction in any medium or format, as long as you give appropriate credit to the original author(s) and the source, provide a link to the Creative Commons licence, and indicate if changes were made. The images or other third party material in this article are included in the article's Creative Commons licence, unless indicated otherwise in a credit line to the material. If material is not included in the article's Creative Commons licence and your intended use is not permitted by statutory regulation or exceeds the permitted use, you will need to obtain permission directly from the copyright holder. To view a copy of this licence, visit http://creativecomm ons.org/licenses/by/4.0/.

Funded by SCOAP ${ }^{3}$.

\section{References}

1. J.M. Bardeen, W.H. Press, S.A. Teukolsky, Rotating black holes: locally nonrotating frames, energy extraction, and scalar synchrotron radiation. Astrophys. J. 178, 347 (1972)

2. S. Chandrasekhar, The Mathematical Theory of Black Holes (Oxford University Press, New York, 1983)

3. C.J. Goebel, Comments on the "vibrations" of a black hole. Astrophys. J. 172, L 95195 (1972)

4. E. Teo, Spherical photon orbits around a Kerr black hole. Gener. Relativ. Gravit. 35, 1909 (2003)

5. P.V.P. Cunha, C.A.R. Herdeiro, Eugen Radu, Fundamental photon orbits: black hole shadows and spacetime instabilities. Phys. Rev. D 96(2), 024039 (2017)

6. J. Grover, Alexander Wittig, Black hole shadows and invariant phase space structures. Phys. Rev. D 96(2), 024045 (2017)

7. P.V.P. Cunha, C.A.R. Herdeiro, M.J. Rodriguez, Does the black hole shadow probe the event horizon geometry? Phys. Rev. D 97(8), 084020 (2018)
8. S.L. Shapiro, S.A. Teukolsky, Black Holes, White Dwarfs, and Neutron Stars: the Physics of Compact Objects (Wiley, New York, 1983), p. 645p

9. V. Cardoso, A.S. Miranda, E. Berti, H. Witek, V.T. Zanchin, Geodesic stability, Lyapunov exponents and quasinormal modes. Phys. Rev. D 79, 064016 (2009)

10. S. Hod, Spherical null geodesics of rotating Kerr black holes. Phys. Lett. B 718, 1552 (2013)

11. Emanuel Gallo, J.R. Villanueva, Photon spheres in Einstein and Einstein-Gauss-Bonnet theories and circular null geodesics in axially-symmetric spacetimes. Phys. Rev. D 92(6), 064048 (2015)

12. Z. Stuchlik, J. Schee, B. Toshmatov, J. Hladik, J. Novotny, Gravitational instability of polytropic spheres containing region of trapped null geodesics: a possible explanation of central supermassive black holes in galactic halos. JCAP 1706(06), 056 (2017)

13. Z. Stuchlik, S. Hledik, Jan Novotny, General relativistic polytropes with a repulsive cosmological constant. Phys. Rev. D 94(10), 103513 (2016)

14. IZh Stefanov, S.S. Yazadjiev, Galin G. Gyulchev, Connection between black-hole quasinormal modes and lensing in the strong deflection limit. Phys. Rev. Lett. 104, 251103 (2010)

15. D. Núñez, H. Quevedo, D. Sudarsky, Black holes have no short hair. Phys. Rev. Lett. 76, 571 (1996)

16. Shahar Hod, A no-short scalar hair theorem for rotating Kerr black holes. Class. Quant. Gravit. 33, 114001 (2016)

17. S. Hod, Hairy black holes and null circular geodesics. Phys. Rev. D 84, 124030 (2011)

18. Y.S. Myung, Hairy mass bound in the Einstein-Born-Infeld black hole. Phys. Rev. D 86, 084047 (2012)

19. Yan Peng, Hair mass bound in the black hole with nonzero cosmological constants. Phys. Rev. D 98, 104041 (2018)

20. Peng, Y., Hair distributions in noncommutative Einstein-BornInfeld black holes. arXiv: 1808.07988

21. S. Hod, The fastest way to circle a black hole. Phys. Rev. D 84, 104024 (2011)

22. S. Hod, Fermat's principle in black-hole spacetimes. Int. J. Mod. Phys. D 27(14), 1847025 (2018)

23. Peng, Y., The shortest orbital period in scalar hairy kerr black holes. arXiv:1901.02601 [gr-qc]

24. J. Keir, Slowly decaying waves on spherically symmetric spacetimes and ultracompact neutron stars. Class. Quant. Gravity 33, 135009 (2016)

25. V. Cardoso, A.S. Miranda, E. Berti, H. Witek, V.T. Zanchin, Geodesic stability, Lyapunov exponents and quasinormal modes. Phys. Rev. D 79, 064016 (2009)

26. S. Hod, Upper bound on the radii of black-hole photonspheres. Phys. Lett. B 727, 345 (2013)

27. P.V.P. Cunha, E. Berti, C.A.R. Herdeiro, Light-Ring Stability for Ultracompact Objects. Phys. Rev. Lett. 119, 251102 (2017)

28. S. Hod, On the number of light rings in curved spacetimes of ultracompact objects. Phys. Lett. B 776, 1 (2018)

29. S. Hod, Upper bound on the gravitational masses of stable spatially regular charged compact objects. Phys. Rev. D 98, 064014 (2018)

30. Yan Peng, On instabilities of scalar hairy regular compact reflecting stars. JHEP 1810, 185 (2018)

31. B. Mashhoon, Stability of charged rotating black holes in the Eikonal approximation. Phys. Rev. D 31(2), 290-293 (1985)

32. S. Hod, Universal bound on dynamical relaxation times and blackhole quasinormal ringing. Phys. Rev. D 75, 064013 (2007)

33. S. Hod, Black-hole quasinormal resonances: wave analysis versus a geometric-optics approximation. Phys. Rev. D 80, 064004 (2009)

34. S.R. Dolan, The quasinormal mode spectrum of a Kerr black hole in the Eikonal limit. Phys. Rev. D 82, 104003 (2010)

35. Y. Decanini, A. Folacci, Bernard Raffaelli, Unstable circular null geodesics of static spherically symmetric black holes, Regge poles and quasinormal frequencies. Phys. Rev. D 81, 104039 (2010) 
36. Y. Decanini, A. Folacci, Bernard Raffaelli, Resonance and absorption spectra of the Schwarzschild black hole for massive scalar perturbations: a complex angular momentum analysis. Phys. Rev. D 84, 084035 (2011)

37. S. Hod, Resonance spectrum of near-extremal Kerr black holes in the eikonal limit. Phys. Lett. B 715, 348-351 (2012)

38. H. Yang, D.A. Nichols, F. Zhang, A. Zimmerman, Z. Zhang, Yanbei Chen, Quasinormal-mode spectrum of Kerr black holes and its geometric interpretation. Phys. Rev. D 86, 104006 (2012)

39. J. Novotný, J. Hladík, Z. Stuchlík, Polytropic spheres containing regions of trapped null geodesics. Phys. Rev. D 95(4), 043009 (2017)
40. Shahar Hod, Analytic study of self-gravitating polytropic spheres with light rings. Eur. Phys. J. C 78(5), 417 (2018)

41. S. Hod, Self-gravitating field configurations: the role of the energymomentum trace. Phys. Lett. B 739, 383 (2014)

42. S. Hod, Lower bound on the compactness of isotropic ultracompact objects. Phys. Rev. D 97, 084018 (2018)

43. H. Bondi, Anisotropic spheres in general relativity. Mon. Not. R. Astron. Soc. 259, 365 (1992) 